positive biopsy cases. A proportion of patients will not have serological tests going straight to endoscopy as first line investigation for their anaemia. Serological testing remains useful in primary care and for physicians to diagnose coeliac disease; however it is important to be aware of the small number of cases (approximately $5 \%$ ) that will be missed when relying on serology alone.

Competing interests None declared.

\section{PWE-123 RESPONSE TO BILE ACID SEQUESTRANTS IS POOR IN PATIENTS WITH EQUIVOCAL SEHCAT RESULTS}

doi:10.1136/gutjnl-2012-302514d.123

${ }^{1} 0$ Orekoya, ${ }^{2}{ }^{2} \mathrm{~J}$ McLaughlin, ${ }^{3} \mathrm{E}$ Leitao, ${ }^{3} \mathrm{~W}$ Johns, ${ }^{2} \mathrm{P}$ Paine. ${ }^{1}$ University of Manchester, Salford Royal Foundation Trust, Manchester, UK; ${ }^{2}$ Department of Gastroenterology, Salford Royal Foundation Trust, Manchester, UK; ${ }^{3}$ Department of Nuclear Medicine, Salford Royal Foundation Trust, Manchester, UK

Introduction Bile acid malabsorption (BAM) is a common cause of chronic diarrhoea that can be diagnosed by the SeHCAT test and treated with bile acid sequestrants (BAS). The purpose of this study was to clarify the use and efficacy of BAS in the treatment of patients with diarrhoea and equivocal SeHCAT results.

Methods Case records were reviewed over a 6-year period for patients investigated by SeHCAT with a positive ( $\leq 8 \%$ ), equivocal $(>8 \%$ and $<16 \%)$ or negative $(>16 \%)$ retention result. Patients were sub-characterised into the following groups. Group 1: terminal ileum Crohn's disease, (pre or post resection) $n=51$. Group 2: diarrhoea predominant irritable bowel syndrome (D-IBS) $n=159$. Group 3: BAM associated with other gastrointestinal disease $n=51$; of which cholecystectomy $(n=37)$, coeliac disease $(n=1)$, chronic pancreatitis $(n=1)$, bacterial overgrowth $(n=2)$, diabetes $(n=4)$ and other gastrointestinal surgeries $(n=6)$. Group 4: terminal ileum disease plus cholecystectomy $n=3$. Patients' sex and age were recorded. Use of BAS (colestyramine or colesevelam) and response were noted.

Results SeHCAT tests were performed in 264 patients and $39(15 \%)$ patients were found to have equivocal results while 104 (39\%) had positive results. Although 28/39 (72\%) patients with equivocal results were offered treatment with BAS, information on response to treatment was only available in half of these patients $(n=14)$. In comparison, there was a higher rate (75\%) of follow-up in the patients with positive SeHCAT results with information on response to treatment being available in 73 of the 97 patients offered BAS treatment. There was a marked difference in response to BAS therapy between the two groups. A successful response was noted in only $36 \%(n=5)$ of patients with equivocal SeHCAT results while $66 \%$ $(n=48)$ of patients with positive SeHCAT results had a successful response. The difference in treatment response was also most significant among the patients in group 2 with D-IBS. 73\% $(n=24 / 33)$ of the patients with positive SeHCAT results in group 2 responded to BAS therapy while only $33 \%(n=3 / 9)$ of those with equivocal SeHCAT results in this same group had a successful response.

Conclusion This retrospective study indicates that there is a poorer response to bile acid sequestrants among patients with equivocal SeHCAT results, however it is possible there was a disproportionate number of non-responders attending for follow-up in this group. More comprehensive follow-up is needed in patients with equivocal SeHCAT results in the future to help determine whether BAS treatment in this lower response group is cost-effective.

Competing interests None declared.

\section{PWE-124 COLESEVELAM USE AND EFFICACY FOR BILE ACID MALABSORPTION}

doi:10.1136/gutjnl-2012-302514d.124

${ }^{1} 0$ Orekoya, ${ }^{2}{ }^{2} \mathrm{~J}$ McLaughlin, ${ }^{3} \mathrm{E}$ Leitao, ${ }^{3} \mathrm{~W}$ Johns, ${ }^{2} \mathrm{P}$ Paine. ${ }^{1}$ University of Manchester, Salford Royal Foundation Trust, Manchester, UK; ${ }^{2}$ Department of
Gastroenterology, Salford Royal Foundation Trust, Manchester, UK; ${ }^{3}$ Department of Nuclear Medicine, Salford Royal Foundation Trust, Manchester, UK

Introduction Bile acid malabsorption (BAM) is a common cause of chronic diarrhoea that can be diagnosed by the SeHCAT test and treated with bile acid sequestrants (BAS). Colestyramine, the most commonly used BAS, is often poorly tolerated due to side effects including nausea, vomiting, flatulence and abdominal pain. Colesevelam, has recently been advocated, as a second line BAS therapy in patients who poorly tolerate colestyramine. ${ }^{1}$ The purpose of this retrospective study was to determine the current use and efficacy of colesevelam in bile acid malabsorption.

Methods Case records were reviewed over a 6-year period for patients found to have a positive SeHCAT test (defined as retention $\leq 8 \%$ ). The age and sex, indication for SeHCAT test, use of BAS and clinical response were noted.

Results SeHCAT tests were performed in 264 patients, of which a positive SeHCAT was found in 104 (39\%). Data on use and response to BAS were found in 73 . The majority $(n=68)$ were given colestyramine as first line treatment with only five receiving colesevelam first line. Symptom improvement with colestyramine occurred in $41 / 68$ (60\%). 27/68 (40\%) failed colestyramine therapy of which $2 / 3$ were due to poor tolerance. 12 of these were then offered second line therapy with colesevelam. $42 \%$ of the 12 patients $(n=5)$ who were given colesevelam after failing to respond to or tolerate colestyramine had a positive response to colesevelam second line. None of the patients reported poor tolerance to colesvelam. Overall BAS response was slightly higher among male patients $(76 \%$ success in males vs $60 \%$ success in females) but there were no differences between different age groups.

Conclusion This retrospective study indicates a good response rate and good tolerance to colesevelam in colestyramine non-responders; however its use as second line therapy was low for reasons that are unclear. Further study is needed to establish whether colesevelam might have better efficacy than colestyramine as first line therapy and to raise awareness of its availability.

Competing interests None declared.

\section{REFERENCE}

1. Wedlake L, A'Hern R, Russell D, et al. Systematic review: the prevalence of idiopathic bile acid malabsorption as diagnosed by SeHCAT scanning in patients with diarrhoea-predominant irritable bowel syndrome. Aliment Pharmacol Ther 2009; 30:707-17.

\section{PWE-125 DOES THE TNM STAGING CRITERIA PREDICT SURVIVAL IN PATIENTS WITH SMALL BOWEL NEUROENDOCRINE TUMOURS?}

doi:10.1136/gutjnl-2012-302514d.125

${ }^{1,2} \mathrm{R}$ Srirajaskanthan, ${ }^{*}{ }^{2} \mathrm{~A}$ Ahmed, ${ }^{2} \mathrm{~J} \mathrm{~K}$ Ramage. ${ }^{1}$ Department of Gastroenterology, University Hospital Lewisham, London, UK; ${ }^{2}$ Institute of Liver studies, Kings College Hospital, London, UK

Introduction Small bowel neuroendocrine tumours (SBNETs) are regarded as relatively indolent cancers. A TNM staging system designed by European NET Society (ENETS) was designed to help stage these tumours to enable ease in classification of these tumours. ${ }^{1}$ This study aims to demonstrate whether the TNM stage and grade of tumour predicts survival in this cohort of patients. The cause of death is also analysed.

Aim To retrospectively stage patients with known small bowel primary NETs and see whether survival is dependent on stage and grade of disease. The cause of death in patients with small bowel NETs was also analysed.

Methods A total of 138 patients with SBNETs were identified. Primary site: Duodenal 2.1\% (3), Jejunal 2.9\% (4), ileal 95\% (131). 
Patients with radiologically, endoscopically or surgically proven SBNETs were included in this study, patients with unknown primary were excluded. A total of 623 patient year's follow-up, with a mean duration of follow-up of 5 years. The median age 61 years (range 24-84). Statistical analysis was performed using GraphPad Prism 5.1.

Results TNM staging and follow-up data were available in 118 cases. Due to low numbers of Stage 2 and 3 tumours these were group together for comparison. There were four cases with stage 2 , 23 cases with Stage 3 and 91 cases with stage 4 small bowel NETs. Kaplan-Meier plots were constructed these demonstrated a significant difference in survival between patients with different stage of disease $(p=0.03)$. There was no significant difference in survival between stage 2 and stage 3 diseases. There was a significant survival difference between G1 (Ki67 $\leq 2)$ vs G2 (Ki67 3-20) $\mathrm{p}=0.049$. The overall 5 -year and 10 -year survival was $79.5 \%$ and $48.5 \%$ respectively for all patients independent of stage of disease. Of the patients that died the median time to death from diagnosis was 3 years (range $0-14$ ). The cause of death was related to tumour burden in 50\% (22 patients), carcinoid heart disease in 11.3\% (five patients), post intervention (one case surgery, one case post-embolisation) $4.5 \%$, small bowel obstruction or perforation $13.6 \%$ (six patients) and non-tumour related deaths in $24.5 \%$ (9) patients.

Conclusion This study demonstrates the overall 5-year and 10-year survival is higher than that published in the SEER data. ${ }^{2}$ The cause of death demonstrates the non-tumour or disease related deaths account for $24.5 \%$ of cases. There is significant survival difference between Stage IV disease and Stage II and III. There was no significant difference in survival between stage II or III. Low grade tumours Ki67 $\leq 2 \%$ was associated with better survival than Ki67 3-20. No patients had a Ki67 >20; therefore no analysis could be performed.

Competing interests None declared.

\section{REFERENCES}

1. Rindi G, Klöppel G, Couvelard A, et al. TNM staging of midgut and hindgut (neuro) endocrine tumors: a consensus proposal including a grading system. Virchows Arch 2007; 451:757-62.

2. Yao JC, Hassan M, Phan A, et al. One hundred years after "carcinoid": epidemiology of and prognostic factors for neuroendocrine tumors in 35,825 cases in the United States. J Clin Oncol 2008:26:3063-72.

\section{PWE-126 SURVIVAL AND RECURRENT DISEASE IN PATIENTS WITH RESECTED PRIMARY SMALL BOWEL NEUROENDOCRINE TUMOURS}

doi:10.1136/gutjnl-2012-302514d.126

\begin{abstract}
${ }^{1,2} \mathrm{R}$ Srirajaskanthan, ${ }^{*} \mathrm{~A}$ Ahmed, ${ }^{2} \mathrm{~J} \mathrm{~K}$ Ramage. ${ }^{1}$ Department of Gastroenterology, University Hospital Lewisham, London, UK; ${ }^{2}$ Institute of Liver studies, Kings College Hospital, London, UK
\end{abstract}

Introduction Small bowel neuroendocrine tumours (SBNETS) are the most common of all GI NETs. The majority of patients present with metastatic disease. It is unclear whether resection of the primary tumour improves prognosis. Furthermore, the recurrence rate of disease in patients following "curative" resection is not previously been investigated.

Aims To demonstrate if primary SBNET resection leads to improved survival and time to development of recurrent disease in patients following resection of primary tumour $+/-$ mesenteric disease in an attempted curative resection.

Methods 138 patients with SBNETs seen in our institution; median duration of follow-up was 5 years. Median age 61 (range 24-84 years). Only patients in whom current disease state was known were included in the study. Primary site: Duodenal 2.1\% (3),
Jejunal 2.9\% (4), ileal 95\% (131). Kaplan-Meier plots were constructed to determine survival. Staging was performed retrospectively using the TNM staging system proposed by ENETs. ${ }^{1}$

Results 100 patients had the primary resected, four patients had irresectable disease at laparotomy. The mean time to resection of primary from diagnosis was 5.8 months (range 0-78 months). There were no deaths within 30 days post surgery. Kaplan-Meier survival curves were constructed. There was a significant survival benefit in patients whom underwent resection of primary tumour compared to those who did not have the primary resected (120 vs 56 months, $\mathrm{p}<0.005)$. There were four patients with Stage 2, 23 patients with stage 3 disease and 91 with stage 4 disease. There were 10 patients in whom it was not possible to accurately stage of disease since the complete histology was not available, however, all of these patients had no evidence of recurrent disease in the initial post-operative period. No survival data were available for the remaining 10 patients. Of the patients who underwent attempted curative resection without distal metastatic disease at presentation, there were 36 patients suitable for analysis. Of these 15 of 36 (41.7\%) patients have developed recurrent disease. Median period for development of recurrence was 55 months (range 11-606 months). There was no recurrence in the four patients with known stage 2 disease (4-168 months). Recurrence occurred in 8 of 23 patients (34.8\%) with stage 3 disease.

Conclusion This study demonstrated a marked improvement in survival in patients who underwent resection of the primary tumour. Disease recurrence is common in patients following curative resection of locally advanced small bowel NETs. Surveillance for a period of only 5 years will not identify a number of patients who will proceed to develop recurrence.

Competing interests None declared.

\section{REFERENCE}

1. Rindi G, Klöppel G, Couvelard A, et al. TNM staging of midgut and hindgut (neuro) endocrine tumors: a consensus proposal including a grading system. Virchows Arch 2007; 451:757-62

\section{PWE-127 TWO RANDOMISED, DOUBLE-BLIND, PLACEBO- CONTROLLED PHASE 3 TRIALS OF LINACLOTIDE IN ADULTS WITH IRRITABLE BOWEL SYNDROME: EFFECTS ON OUALITY OF LIFE}

doi:10.1136/gutjnl-2012-302514d.127

${ }^{1} \mathrm{R}$ T Carson, ${ }^{*}{ }^{1} \mathrm{~S}$ Tourkodimitris, ${ }^{2} \mathrm{~B}$ E Lewis, ${ }^{2} \mathrm{~J} \mathrm{M}$ Johnston. ${ }^{1}$ Forest Research Institute, Jersey City, New Jersey, USA; ${ }^{2}$ rronwood Pharmaceuticals, Cambridge, USA

Introduction Linaclotide, a minimally absorbed guanylate cyclase-C receptor agonist, is an investigational drug for the treatment of irritable bowel syndrome with constipation (IBS-C) that has shown statistically significant improvements in abdominal and bowel symptoms in two Phase 3 clinical trials. IBS-C is a common functional gastrointestinal disorder that affects a significant portion of the population and leads to reduced quality of life (QOL).

Methods Data evaluating the efficacy and safety of oral once-daily linaclotide $290 \mu \mathrm{g}(\mathrm{N}=748)$ vs placebo $(\mathrm{N}=742)$ in patients with IBS-C were pooled from two Phase 3 clinical trials. Patients meeting modified Rome II criteria for IBS-C were randomised to receive either linaclotide or placebo treatment for 12 weeks. The Irritable Bowel Syndrome Quality of Life (IBS-QOL) questionnaire, comprising 34 items, each with a 5 -point response scale $(1=$ "not at all" through to $5=$ "extremely" or "a great deal"), was completed at baseline and also at the end of the treatment period. The IBS-QOL is scored "overall" and by eight subscales (Dysphoria, Interference with Activity, Body Image, Health Worry, Food Avoidance, Social Reaction, Sexual and Relationships). The change from baseline to week 